\title{
A retrospective study evaluating the efficacy of identification and management of sepsis at a district-level hospital internal medicine department in the Western Cape Province, South Africa, in comparison with the guidelines stipulated in the 2012 Surviving Sepsis Campaign
} \author{
A H Oosthuizen, ${ }^{2} \mathrm{MB}$ ChB (Cum Laude), Dip PEC (SA), MMed EM, FCEM (SA) \\ ${ }^{1}$ Department of Internal Medicine, Karl Bremer Hospital, Cape Town, South Africa \\ ${ }^{2}$ Department of Emergency Medicine, Karl Bremer Hospital, Cape Town, South Africa
}

R Bhikoo, ${ }^{1}$ MB ChB; S Versfeld, ${ }^{1}$ MB ChB; M M De V Basson, ${ }^{1}$ MB ChB, Hons BSc (Epidemiol), MMed (Int Med);

Corresponding author: R Bhikoo (raisa3121@gmail.com)

\begin{abstract}
Background. Currently there is little information on the identification, management and outcomes of patients with sepsis in developing countries. Simple cost-effective measures such as accurate identification of patients with sepsis and early antibiotic administration are achievable targets, within reach without having to make use of unsustainable protocols constructed in developed countries.

Objectives. To assess the ability of clinicians at a district-level hospital to identify and manage sepsis, and to assess patient outcome in terms of in-hospital mortality and length of hospital stay given the above management.

Methods. A retrospective descriptive study design was used, analysing data from the routine burden of disease audit done on a 3-monthly basis at Karl Bremer Hospital (KBH) in the Western Cape Province, South Africa.

Results. The total sample size obtained was 70 patients, of whom 18 (25.7\%) had an initial triage blood pressure indicative of sepsis-induced hypotension. However, only 1 (5.5\%) of these 18 patients received an initial crystalloid fluid bolus of at least $30 \mathrm{~mL} / \mathrm{kg}$. The median time that elapsed before administration of antibiotics in septic shock was 4.25 hours. Furthermore, a positive delay in antibiotic administration ( $p=0.0039)$ was demonstrated. The data also showed that $8 / 12$ patients $(66.7 \%)$ with septic shock received inappropriate amounts of fluids. The in-hospital mortality rate for sepsis was $4 / 24$ (16.7\%), for severe sepsis 11/34 (32.3\%) and for septic shock a staggering 9/12 (75.0\%). Conclusions. The initial classification process and management of sepsis by clinicians at $\mathrm{KBH}$ is flawed. This inevitably leads to an increase in in-hospital mortality.
\end{abstract}

S Afr Med J 2017;107(8):674-678. DOI:10.7196/SAMJ.2017.v107i8.11019

Sepsis, as defined by the Surviving Sepsis Campaign (SSC) in 2012, ${ }^{[1]}$ is the presence of a probable or documented infection together with systemic manifestations of an infection.

In 2004, the World Health Organization ${ }^{[2]}$ listed three infective causes, namely lower respiratory tract infection, diarrhoeal disease and tuberculosis, on their top ten list of causes of death, which is similar to mortality reports documented in the 2013 Western Cape Mortality Profile. ${ }^{[3]}$ This highlights the fact that sepsis and its sequelae - which were originally caused by an infection - are major contributors to the local and global burden of disease.

To date there are virtually no accurate data on the incidence and prevalence of or mortality rates for sepsis, severe sepsis and septic shock in developing countries. Mortality rates have been reported to be as high as $30 \%$ for sepsis, $40 \%$ for severe sepsis and $80 \%$ for septic shock $^{[4-6]}$ in developed countries. Septic shock is still the leading cause of death in intensive care units worldwide. ${ }^{[5]}$ Data from developed countries show a continuous increase in the incidence of sepsis, further emphasising the need to review management protocols in order to reduce morbidity and mortality.

In developed countries, the implementation of protocols for the identification of sepsis and for its management have contributed to a decline in mortality rates. ${ }^{[7]}$ In low-income countries, prob- lems such as access to healthcare, cost constraints, lack of resources and delayed presentation of patients with sepsis make it difficult to implement protocols based on the patient profile of developed countries. In sub-Saharan Africa there has therefore been a widespread shift towards the development of cost-effective protocols specifically suited to the local epidemiological and ecological characteristics. ${ }^{[8,9]}$ In 2006, a Ugandan-based prospective study assessed the management and outcomes of hospitalised patients with severe sepsis syndromes. Approximately $85 \%$ of their sample were HIV-positive. Factors contributing to mortality included inadequate fluid administration, lack of uniformity in administration of antibiotics appropriate to the source of sepsis, and delay in antibiotic administration. ${ }^{[8]} \mathrm{A}$ follow-up prospective study was done in 2008 using the previous study as the observation cohort. Interventions included early appropriate antibiotic and intravenous fluid administration. Mortality at 30 days was significantly lower in the intervention cohort compared with the observation cohort, leading to the conclusion that simple and inexpensive management can improve outcome. ${ }^{[8,9]}$

Currently no data are available regarding sepsis management in our unique setting in South Africa (SA). We hope to pilot the way for further research in this field. 


\section{Objective}

The primary objective was to determine the efficacy of sepsis identification and management by clinicians at Karl Bremer Hospital $(\mathrm{KBH})$, a large district-level hospital in Cape Town, SA. Furthermore, we aimed to determine the demographics of patients presenting with sepsis, the burden of sepsis and its management, and mortality rates and length of hospital stay among patients admitted. Key determinants of efficacy were assessed using guidelines outlined in the $\mathrm{SSC}^{[1]}$ as a means of comparison. The SSC, initially published in 2004, reviewed data on the management of severe sepsis and septic shock. The recommendations are intended to be best practice and not standard of care.

\section{Methods}

\section{Setting}

$\mathrm{KBH}$ is a large district-level hospital with a total of 310 beds, servicing an average of 11000 patients per month. The estimated total cost per patient per day is ZAR2 105.90 for a ward admission. However, 72\% of the patients are classified as earning either no income or $<$ ZAR3 000 per month, and are therefore not obliged to pay the full fee. A fourbed high-care unit shared by all the departments is able to manage ventilated patients. However, if a prolonged high-care admission is anticipated, the patient will need to be transferred to a fully equipped intensive care unit managed by a dedicated team at a tertiary facility

\section{Sepsis}

Widespread systemic manifestations due to the existence of an infection. The systemic manifestations could be any of the following (adapted from SSC ${ }^{(1)}$ ):

\section{General variables:}

- Fever $\left(>38.3^{\circ} \mathrm{C}\right)$

- Hypothermia (core temperature $<36^{\circ} \mathrm{C}$ )

- Heart rate $>90 \mathrm{bpm}$

- Tachypnoea, respiratory rate $>25 / \mathrm{min}$

- Altered mental status

- Significant oedema or positive fluid balance $(>20 \mathrm{~mL} / \mathrm{kg}$ over $24 \mathrm{~h}$ )

- Hyperglycaemia (plasma glucose $>140 \mathrm{mg} / \mathrm{dL}$ or $7.7 \mathrm{mmol} / \mathrm{L}$ ) in the absence of diabetes

Inflammatory variables:

- Leukocytosis (white cell count $>10 \times 10^{9} / \mathrm{L}$ ) or leukopenia (white cell count $<4 \times 10^{\circ} / \mathrm{L}$ )

- Plasma C-reactive protein $>4 \mathrm{mg} / \mathrm{L}$

Haemodynamic variables:

- Arterial hypotension (systolic blood pressure $<90 \mathrm{mmHg}$, mean arterial pressure $<70 \mathrm{mmHg}$ )

Organ dysfunction variables:

- Arterial hypoxaemia $\left(\mathrm{PaO}_{2} / \mathrm{FiO}_{2}\right.$ ratio $\left.<300\right)$

- Acute oliguria (urine output $<0.5 \mathrm{~mL} / \mathrm{kg} / \mathrm{h}$ for at least $2 \mathrm{~h}$ despite adequate fluid resuscitation)

- Raised creatinine ( $>44.2 \mu \mathrm{mol} / \mathrm{L})$

- Coagulation abnormalities (INR $>1.5$ or aPTT $>60 \mathrm{~s}$ )

- lleus (absent bowel sounds)

- Thrombocytopenia (platelet count $<100 \times 10^{9} / \mathrm{L}$ )

- Hyperbilirubinaemia (plasma total bilirubin $>22 \mu \mathrm{mol} / \mathrm{L}$ )

Tissue perfusion variables:

- Hyperlactataemia (>1 mmol/L)

- Decreased capillary refill or mottling such as the nearby Tygerberg Hospital. Additionally, the emergency department (ED) at KBH has three resuscitation beds.

\section{Study design}

A retrospective descriptive study design was used. The internal medicine department at $\mathrm{KBH}$ performs a routine burden of disease audit on a 3-monthly basis. Data for the audit are obtained from triplicate discharge letters, hospital transfer notes and death certification summaries containing all relevant information on the patient's hospital stay, from admission to discharge or death. Data from the burden of disease audit for November 2015 - January 2016 were reviewed. For a patient to be selected for the study, the data from the audit sheet had to state any one of the key words 'sepsis', 'severe sepsis' or 'septic shock' as the diagnosis at discharge or death. The suspected or confirmed infection had to have been present at ED presentation for the patient to qualify for the study. Data were assessed using a data collection sheet compiled by the authors that focused specifically on clinical and blood gas measurements that could be used in the ED to classify patients as having sepsis, severe sepsis or septic shock. Time to first-dose antibiotics (grade $1 \mathrm{~B}$ evidence based on the $\mathrm{SSC}^{[1]}$ ), source-appropriate antibiotics (grade 1B), amount of intravenous fluids (grade 1C) and arterial blood gas/lactate measurement were also assessed.

Once the data were obtained, we retrospectively classified patients into the sepsis, severe sepsis or septic shock groups based on the

\section{Severe sepsis}

Sepsis PLUS acute organ dysfunction or tissue hypoperfusion.

Sepsis-induced hypotension:

- Systolic blood pressure $<90 \mathrm{mmHg}$, or

- Mean arterial pressure $<70 \mathrm{mmHg}$, or

- Systolic blood pressure decrease $>40 \mathrm{mmHg}$

Organ dysfunction variables:

- Arterial hypoxaemia $\left(\mathrm{PaO}_{2} / \mathrm{FiO}_{2}\right.$ ratio $\left.<300\right)$

- Acute oliguria (urine output $<0.5 \mathrm{~mL} / \mathrm{kg} / \mathrm{h}$ for at least $2 \mathrm{~h}$ despite adequate fluid resuscitation)

- Raised creatinine (>45 $\mu \mathrm{mol} / \mathrm{L}$ )

- Coagulation abnormalities (INR >1.5 or aPTT >60 s)

- lleus (absent bowel sounds)

- Thrombocytopenia (platelet count $<100 \times 10^{\circ} / \mathrm{L}$ )

- Hyperbilirubinaemia (plasma total bilirubin $>22 \mu \mathrm{mol} / \mathrm{L}$ )

Tissue perfusion variables:

- Hyperlactataemia (>1 mmol/L)

- Decreased capillary refill or mottling

III. Septic shock

Severe sepsis PLUS evidence of tissue hypoperfusion not reversed with effective fluid resuscitation.

Sepsis-induced tissue hypoperfusion:

Blood lactate concentration $>4 \mathrm{mmol} / \mathrm{L}$

OR

Sepsis-induced hypotension (as above)

Fig. 1. Definitions used for the classification of sepsis. (SSC $=$ Surviving Sepsis Campaign; $\mathrm{PaO}_{2}=$ partial pressure of arterial oxygen; FiO $\mathrm{O}_{2}=$ fractional inspired oxygen; INR = international normalised ratio; aPTT = activated partial thromboplastin time.) 
information that had been available to the casualty doctor at the time of presentation. Definitions used for the classification of sepsis are set out in Fig. 1.

\section{Statistical analysis}

All data collected were captured onto a Microsoft Excel database, version 2013 (Microsoft, USA). Data analysis was done in Microsoft Excel, version 2013, and statistical analysis in Statistica, version 12 (StatSoft, USA). The level of statistical significance was set at $p<0.05$. The sign test was used to compare descriptive variables. To assess for data association, logistical regression testing and negative binomial regression testing were performed, with the data then being presented as odds ratios (ORs) with $95 \%$ confidence intervals (CIs).

\section{Ethics approval}

Ethics approval was obtained from the Health Research Ethics Committee at the Stellenbosch University (ref. no. N15/10/103).

\section{Results}

Of the 1000 patients reviewed in the burden of disease audit, 70 $(7.0 \%)$ were included in the study. Of the 70 patients, $34(48.5 \%)$ were male and $36(51.4 \%)$ were female. The overall mean (standard deviation (SD)) age of patients presenting with a sepsis syndrome was 48 (9.5) years (minimum 17, maximum 85). A total of 25 patients (35.7\%) were HIV-positive. The most common infective cause for sepsis identified by emergency personnel, across all the grades, was a lower respiratory tract infection. Not surprisingly, diarrhoeal disease/ acute gastroenteritis was the second most common diagnosis made (Figs 2 - 4).

Based on the data collection and subsequent sepsis subgrouping, evaluated using the initial information available to the casualty doctor, $24 / 70$ patients (34.2\%) were classified as having sepsis, $34 / 70$ (48.6\%) as having severe sepsis and $12 / 70(17.1 \%)$ as having septic shock. However, 18/24 (75.0\%) of patients with sepsis, 8/34 (23.6\%) with severe sepsis and $3 / 12(25.0 \%)$ with septic shock did not have their arterial blood gas or lactate level measured at initial presentation.

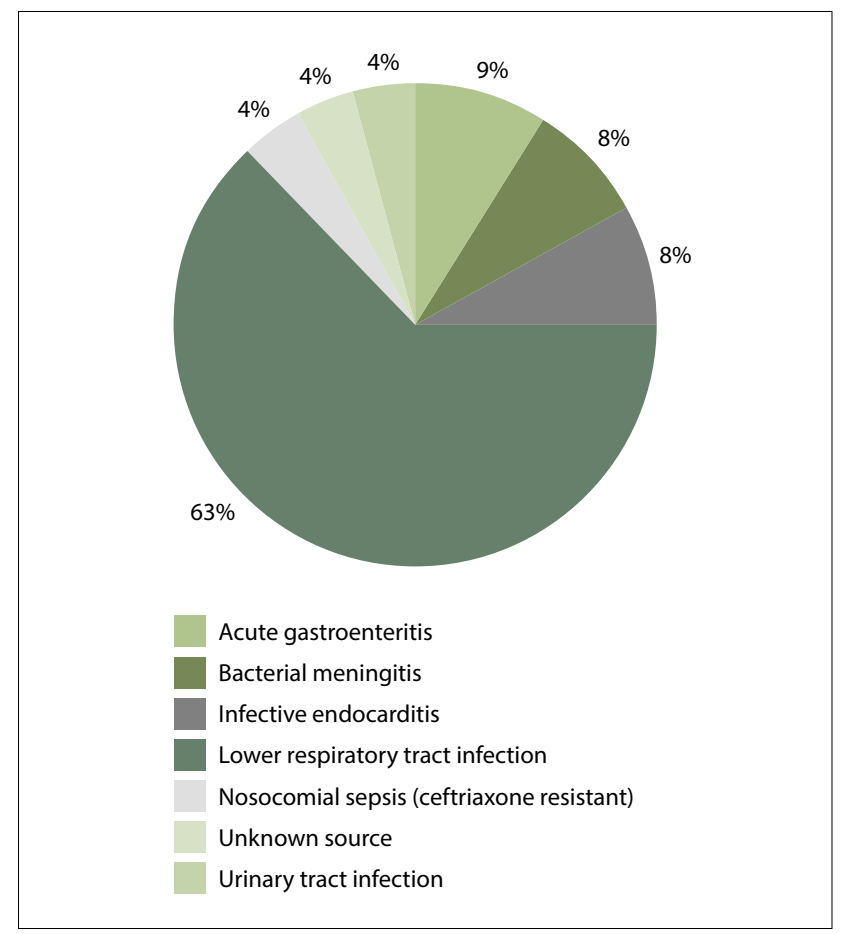

Fig. 2. Suspected sources of sepsis.
Furthermore, no patient who had an initial lactate level measured had a repeat level measured to assess lactate clearance or fluid responsiveness. As a result, many patients who should probably have been classified as having septic shock were classified as having severe sepsis based on adherence to definitions. A total of $18 / 70$ patients (25.7\%) had an initial triage blood pressure indicative of sepsis-induced hypotension, but only $1 / 18$ (5.6\%) of these received an initial crystalloid fluid bolus of at least $30 \mathrm{~mL} / \mathrm{kg}$ and subsequent immediate blood pressure recheck for fluid responsiveness. Furthermore, 6/18 patients (33.3\%) had no initial

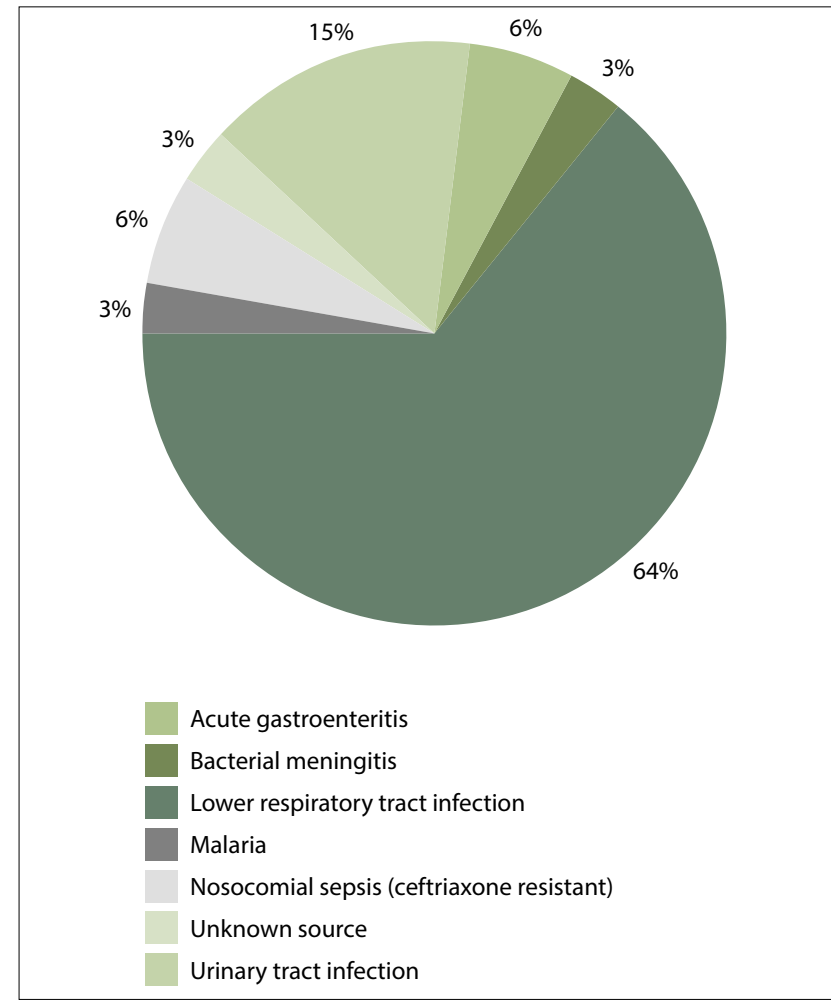

Fig. 3. Suspected sources of severe sepsis.

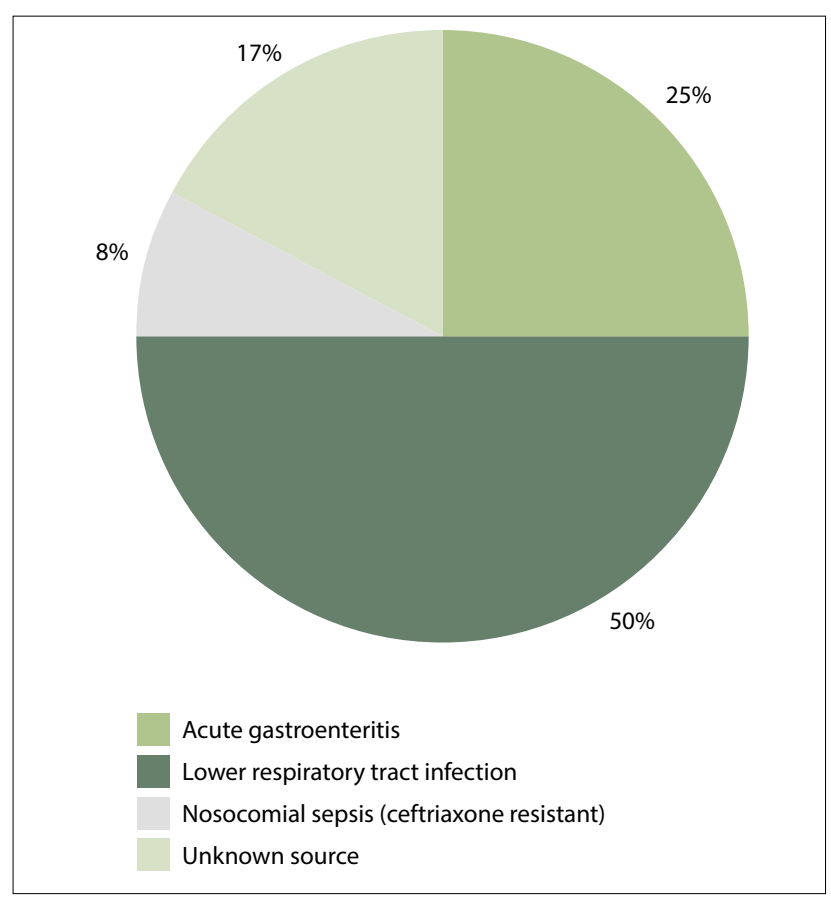

Fig. 4. Suspected sources of septic shock. 


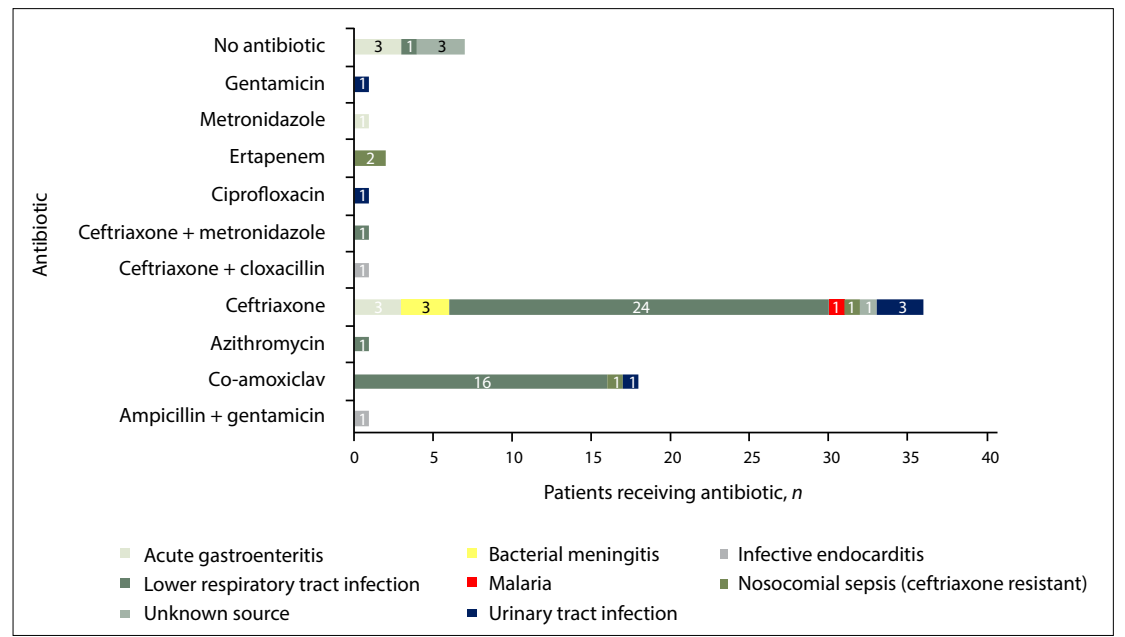

Fig. 5. Antibiotics prescribed during the first 24 hours after presentation.

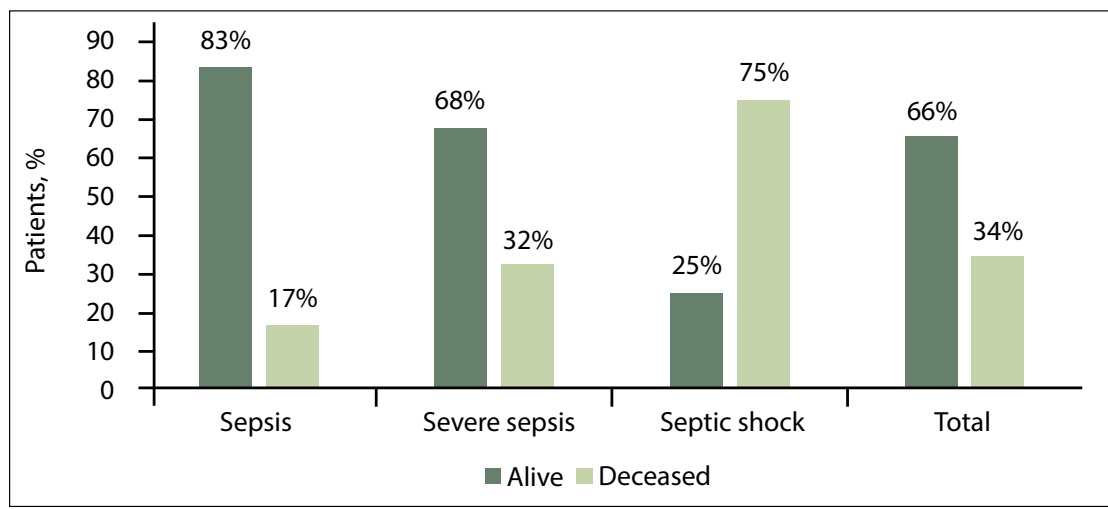

Fig. 6. In-hospital mortality rates for sepsis, severe sepsis and septic shock.

arterial blood gas or lactate level measured, so we had no way of knowing whether the patient was initially classified as having severe sepsis or septic shock, forcing us to assign patients to the severe sepsis group.

The antibiotic most frequently prescribed for sepsis, across all the grades, was ceftriaxone (Fig. 5). Bearing in mind the literature review ${ }^{[1]}$ mentioned above regarding source-appropriate antibiotics, $55 / 70$ patients $(78.5 \%)$ were considered to have received the correct antibiotics for the respective source. However, no HIVpositive patient in the study received cover for Mycobacterium tuberculosis or Pneumocystis jiroveci pneumonia during the first 24 hours after presentation to hospital, despite the clinician having a documented suspicion thereof.

Interestingly, it is also important to note that $7 / 70(10.0 \%)$ of the patients received no antibiotics during the first 24 hours after presentation, despite being identified by the casualty doctor as having a possible infective cause for their clinical symptoms. In these cases, the probable cause of sepsis was equally distributed between acute gastroenteritis and an unknown source. Of these patients, 3/7 $(42.9 \%)$ could be classified as having septic shock based on the initial lactate level.
The median time that elapsed before administration of antibiotics during the first 24 hours after presentation, across all grades of sepsis, was 3.63 hours for sepsis (range $1.67-10.30$ ), 1.58 hours for severe sepsis (range $1.00-2.83$ ) and 4.25 hours for septic shock (range 1.00 - 23.98). Here it is important to note that the above are times from consultation with the attending doctor to antibiotic administration, which depending on how long patients had to wait to see a doctor, may differ considerably from the times from initial presentation at the emergency department to receiving an antibiotic. Unfortunately the latter could not were not documented. To assess whether antibiotics were given within the 3-hour time frame for sepsis and severe sepsis, the sign test was used to extrapolate the data. The results showed that there was no delay in antibiotic administration for sepsis or severe sepsis $(p=0.2706$ for sepsis and $p=0.9997$ for severe sepsis), bearing in mind that the 3-hour mark was used as the cut-off for optimal time to initial antibiotic administration. For septic shock, however, a cut-off of 1 hour was used for optimal time be assessed because initial presenting times to antibiotic administration, and here the sign test showed a positive delay in antibiotic administration ( $p=0.0039$ ).

With regard to the early appropriate intravenous fluid administration discussed above, the data showed that $5 / 24(20.8 \%)$ of patients with sepsis, $8 / 34$ (23.6\%) with severe sepsis and 8/12 (66.7\%) with septic shock received inappropriate amounts of fluids. As mentioned above, only one patient received an initial fluid bolus of at least $30 \mathrm{~mL} / \mathrm{kg}$ for sepsis-induced hypotension. Pearson's $\chi^{2}$ test showed a positive association between septic shock and inappropriate intravenous fluid administration $(p=0.009)$. Furthermore, the proportion of patients with septic shock who received appropriate intravenous fluids was less than half the proportions in the sepsis and severe sepsis groups. As mentioned above, no patient in our study had repeat arterial blood gas measurements to assess lactate clearance, a marker of response to therapy. Also, no invasive measures such as central venous pressures or arterial line blood pressure readings were used to assess fluid responsiveness optimally during the first 24 hours of management.

Overall, the outcome of patients in the study was determined by discharge or in-hospital mortality, the in-hospital mortality rate for sepsis being $4 / 24$ (16.7\%), that for severe sepsis 11/34 (32.3\%) and that for septic shock a staggering $9 / 12$ (75.0\%) (Fig. 6). The mean (SD) age of the patients who died, across all sepsis syndromes, was 53 (9.5) years. The mean length of stay for all sepsis syndromes was 6.3 (2.3) days.

Logistic regression testing to investigate for possible associations between in-hospital mortality rates and various sepsis management principles revealed a positive association between in-hospital mortality and the following:

- Time to first dose of antibiotic (OR 1.07, $95 \%$ CI $1.01-1.14 ; p=0.027$ ). For every 1 hour's delay in antibiotic administration, the chance of death increased by $7 \%$.

- Source-appropriate antibiotics (OR 0.17, $95 \%$ CI 0.05 - 0.59; $p=0.005$ ). The chance of death for patients who received sourceappropriate antibiotics was $83 \%$ less than for those who did not.

- Early appropriate administration of intravenous fluid (OR 0.33, 95\% CI 0.11 - 0.95; $p=0.040$ ). Appropriate intravenous fluids were associated with a $67 \%$ reduction in in-hospital mortality.

\section{Discussion}

The findings reported above reflect clear faults in the identification of sepsis at $\mathrm{KBH}$ and its resultant suboptimal management. 
Evidence to support poor recognition of sepsis syndromes includes lack of adequate fluid boluses for patients who met the definition for hypotension at admission, indicating that clinicians did not recognise the importance of hypotension as a clinical indicator of organ dysfunction in sepsis. Clinicians at KBH do not have access to laboratory results for a minimum of 12 hours after a consultation and are therefore forced to use their clinical judgement regarding the severity of disease, aided by blood pressure monitoring, urine output and blood gas measurement for lactate, and partial pressure of arterial oxygen/fractional inspired oxygen ratios to assess fluid responsiveness and organ dysfunction. Further evidence of inadequate identification of sepsis are the 3/7 patients who could be classified as having septic shock on the basis of the initial blood gas lactate measurement but did not receive antibiotics for the first 24 hours after presentation. This indicates a poor understanding of the effect of delayed antibiotic administration on mortality rates related to sepsis. The overall median time to initial antibiotic administration was 4.25 hours for septic shock v. 1.58 hours for severe sepsis. Because these three patients were included in the count for patients with septic shock, the median time to initial antibiotic administration in this subgroup increased significantly.

Kumar et al.${ }^{[6]}$ showed a $7.6 \%$ decrease in survival for every 1 hour's delay in antibiotic therapy over the ensuing 6 hours. Similarly, we found a $7 \%$ increase in mortality for every hour's delay in antibiotic administration. As mentioned above, the median time to the first dose of antibiotic was measured as the time from consultation with a doctor to initial antibiotic administration. The minimum waiting time in the ED from arrival at the hospital to doctor consultation is 45 - 60 minutes. None of the patients managed, regardless the grade of sepsis, are therefore achieving the targets of the 3-hour bundle and 1-hour administration time frames. This can be attributed to both poor identification, as discussed above, and resource limitations. The nursing staff in the ED who are responsible for drug and fluid administration often oversee the management of $10-20$ patients at a time, which makes it difficult for them to perform important tasks timeously. Bed constraints and lack of resuscitation room availability mean that severely ill patients often lie in the general ED area where there is no appropriate monitoring.

In our study, not receiving source-appropriate antibiotics had a positive association with mortality. Leibovici et al. ${ }^{[10]}$ showed that survival improved when empirical antibiotic treatment matched the in vitro susceptibility of the likely pathogen. The concern in this regard is that we have limited evidence regarding source-appropriate antibiotics in an environment with a high prevalence of HIV infection. Infective aetiologies in sub-Saharan Africa differ from those reported in the SSC guidelines, ${ }^{[1]}$ and in fact certain studies reviewed by the SSC committee excluded HIV-positive patients, giving rise to the questions which are the early source-appropriate antibiotics that should be administered given the unique sub-Saharan African environment, and what the effect on outcome is in HIV-positive patients.

Appropriate intravenous fluid administration is a further area for debate in the HIV-prevalent SA setting. The studies in Uganda and Zambia ${ }^{[8,9,11]}$ reported concerns that in such a setting, large fluid boluses could worsen respiratory failure. However, we found a $67 \%$ reduction in in-hospital mortality with appropriate intravenous fluid administration. Again, lack of appropriate monitoring in the ED makes appropriate fluid administration difficult, as patients receiving large fluid boluses run the risk of becoming fluid overloaded unless they are adequately monitored.

\section{Recommendations}

It is evident that education for all healthcare providers involved in the identification and management of sepsis is necessary. Formulation of a suggested process, perhaps in the form of a sepsis check sheet, could lead to improved management. Re-evaluation of outcomes in the form of length of hospital stay and in-hospital mortality will be necessary in order to evaluate the impact of such a process. Further areas that need attention are earlier availability of laboratory results, which aids decision-making, and more doctors in the ED to decrease waiting time.

\section{Conclusions}

The main aim of our study was to evaluate identification and management of the sepsis syndromes at a district-level hospital in the Western Cape. We concluded that the initial classification of sepsis, severe sepsis and septic shock by our clinicians is flawed. This is largely due to lack of understanding on the part of medical personnel of the clinical evidence needed to support the classification process. This clinical evidence does not depend on delayed laboratory results, but on basic examinations and investigations available in the ED. Priorities in the management of the sepsis syndromes include early source-appropriate antibiotics and early appropriate intravenous fluid administration. These should be the cornerstones of management, and can be instituted regardless of resource availability. Finally, it is evident that additional research is needed in the field of sepsis identification and management in a resource-limited setting. However, basic management principles can nevertheless be implemented, with the potential for an enormous impact on patient survival.

Acknowledgements. Special thanks to Dr Zirkia Joubert and all the internal medicine medical officers at $\mathrm{KBH}$ for granting us the time we needed to work on this study, as well as for all their assistance with patient identification and data collection. We thank Mr M T Chirehwa for the data analysis and his subsequent interpretation of the statistical outcomes. Finally, we thank Mr Iesrafeel Jakoet and Mr Brendon Versfeld for all their help with the write-up and editing of the article.

Author contributions. RB: primary author, literature review, data collection, interpretation and write-up; SV: assisted in data collection and write-up; MMDeVB and AHO: supervisors. All the authors consented to the publication of the article.

Funding. None.

\section{Conflicts of interest. None.}

1. Dellinger RP, Levy MM, Rhodes A, et al. Surviving Sepsis Campaign: International guidelines for

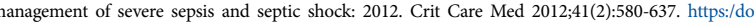
org/10.1097/CCM.0b013e31827e83af

2. World Health Organization. The Global Burden of Disease: 2004 Update. 2008. http://www.who.in healthinfo/global_burden_disease/2004_report_update/en/index.html (accessed 16 February 2017).

3. Morden E, Groenewald P, Zinyakatira N, et al. Western Cape Mortality Profile 2013. Cape Town: SA Medical Research Council, 2016. http://www.mrc.ac.za/bod/WC2013MortalityReport.pdf (accessed 27 June 2017)

Catenacci MH, King K. Severe sepsis and septic shock: Improving outcomes in the emergency department. Emerg Med Clin North Am 2008;26(3):603-623. https:/doi.org/10.1016/j.emc.2008.05.004

5. Degoricija V, Sharma M, Legac A, et al. Survival analysis of 314 episodes of sepsis in medical intensive care unit in university hospital. Impact of intensive care unit performance and antimicrobial therapy Croat Med J 2006:47(3):385-397.

6. Kumar G, Kumar N, Taneja A, et al. Nationwide trends of severe sepsis in the 21st century (2000 - 2007). Chest 2011;140(5):1223-1231. https:/doi.org/10.1378/chest.11-0352

Kaukonen KM, Bailey M, Suzuki S, et al. Mortality related to severe sepsis and septic shock among critically ill patients in Australia and New Zealnd, 2000 - 2012. JAMA 2014:311(13):1308-1316. bron critically ill patients in Australia

8. Jacob ST, Banta P. Bacten JM, et al. The impact of early monitored management on survival in hop hospitalized adult Ugandan patients with severe sepsis. A prospective interver

9. Jacob ST, Moore CC, Banura P, et al. Severe sepsis in two Ugandan hospitals: A prospective observational study of management and outcomes in a predominantly HIV-1 infected population. PLoS One 2009;4(11):e7782. https:/doi.org/10.1371/journal.pone.0007782

10. Leibovici L, Shraga I, Drucker M, et al. The benefit of appropriate empirical antibiotic treatment in patients with bloodstream infection. J Intern Med 1998;244(5):379-386. https:/doi.org/10.1046/j.13652796.1998.00379.

11. Andrews B, Muchemwa L, Kelly P, et al. Simplified severe sepsis protocol: A randomized controlled tria of modified early goal-directed therapy in Zambia. Crit Care Med 2014;42(11):2315-2324. https:/doi org/10.1097/CCM.0000000000000541 